\title{
PERFIL ANTROPOMETRICO E DE APTIDÃO FÍSICA DE IDOSOS PARTICIPANTES DE GRUPOS DE CONVIVÊNCIA
}

Arestides Pereira da Silva Júnior

Maria Luiza de Jesus Miranda

Marilia Velardi

\section{Resumo}

O presente estudo teve como objetivo analisar e comparar o perfil antropométrico e de aptidão física dos participantes dos Grupos de Convivência de Idosos, denominados "Paz e Amor" e "Amizade" do município de Marechal Cândido Rondon-PR. A amostra foi composta por 60 sujeitos, sendo 30 de cada Grupo. A análise dos dados foi realizada através de estatística, útilizando o teste $t$ de Student independente. Os resultados da antropometria demonstraram que ambos os Grupos apresentaram resultados acima dos recomendáveis para a Organização Mundial da Saúde. Os desempenhos dos sujeitos dos dois Grupos nos testes de aptidão física corroboraram com estudos da área. Conclui-se que os Grupos comparados são semelhantes nas variáveis analisadas, provavelmente devido às características gerais da população avaliada.

\section{Palavras-Chave}

Idosos; Antropometria; Aptidão física.

\section{PROFILE ANTROPOMETRIC AND OF PHYSICAL APTITUDE OF ELDERLY COMPANY GROUPS PARTICIPANTS}

Arestides Pereira da Silva Júnior

Maria Luiza de Jesus Miranda

Marina Velardi

\begin{abstract}
The present study aimed to analyze and compare the anthropometrical and physical aptitude profile of the participators of the Elderly Acquaintance Groups, named "Paz e Amor" and "Amizade" from the Municipality of Marechal Candido Rondon - PR. The sample has been compound of 60 individuals, from these individuals 30 were from each group. The data analysis has been carried out through the statistics by using the test $t$ of the independent Student. The results of the anthropometry have shown that both Groups showed results above the recommendable for the Health World Organization. The individuals' performance of both Groups on these physical aptitude tests corroborated with the area studies. It is concluded that the compared Groups are similar on the analyzed variable, probably due to the general characteristics of the evaluated population.
\end{abstract}

\section{Key-Words}

Elderly; Anthropometry; Fitness. 


\section{INTRODUÇÃO}

De acordo com Matsudo (2001) o processo de envelhecimento está associado a mudanças principalmente na estatura, peso e composição corporal, implicando de certa forma ao surgimento de certas doenças, nos níveis de aptidão física e conseqüentemente na capacidade funcional.

Bons níveis de aptidão física em idosos têm se mostrado indicador importante sobre a melhoria de vários aspectos físicos e funcionais, por exemplo, bons parâmetros de composição corporal, controle de peso, capacidade funcional e condicionamento físico melhorado (MATSUDO, 2005; VALE, et. al., 2005; ZAGO; GOBBI, 2003).

Alem disso, serve como parametro de avaliação na elaboração de um programa de Educação física e na auto-avaliação do sujeito frente ao seu estado de condicionamento físico (MATSUDO, 2005).

Rogatto e Gobbi (2001) e Mazo et. al. (2005) afirmam que bons níveis de aptidão física em idosos temse mostrado indicador importante sobre a realização de tarefas do cotidiano, possibilitando, desta forma, que indivíduos sejám independentes fisicamente, alem disso, tem importante papel no aspecto preventivo e aumento da auto-estima. Assim sendo, conhecer os níveis de aptidão física dos indivíduos antes e durante a participação em um programa de atividade física torna-se crucial para que as atividades condigam com as suas potencialidades físicas.

O American College of Sports Medicine (ACSM, 2000) ressalta que medidas de aptidão física são praticas comuns e apropriadas em programas de exercícios preventivos e de reabilitação. Destacam-se os seguintes objetivos de testes de aptidão física em um programa:

- Educar os participantes sobre seu presente estado de aptidão relativa aos padrões adequados, ou sejá, critérios estabelecidos;

- Mostrar dados que são auxiliares no desenvolvimento da prescrição de exercícios para todos os componentes de aptidão;

- Mostrar a avaliação ou declínio pelos participantes através do programa de exercício;

- Motivar os participantes através do estabelecimento de metas razoáveis e alcançáveis de boa aptidão física;

- Reduzir os riscos de doenças. 
Inúmeras pesquisas e estudos publicados ao longo das duas últimas décadas apontam os benefícios físicos, funcionais e fisiológicos que a pratica da atividade física pode proporcionar a população idosa, principalmente como recurso no processo preventivo de doenças decorrentes do processo do envelhecimento (ACSM, 1999; CAPODAGLIO et. al., 2006; ELWARD et. al., 1992; KACZOR et. al., 2006; MATSUDO et. al., 2003; PENEDO e DAHN, 2006; STEWART et. al., 2001; STRUCK e ROSS, 2006).

Alem disso, a pratica da atividade física regular tem sido descrita na literatura como um excelente meio de atenuar a degeneracao provocada pelo processo de envelhecimento Também nos dominios psicologicos e sociais (ACSM, 1999).

Desta forma, o presente estudo teve como objetivo analisar e comparar o perfil antropometrico e de aptidão física dos sujeitos participantes dos Grupos de Convivência de Idosos, denominados "Paz e Amor" e "Amizade" do município de Marechal Candido Rondon - PR.

\section{MÉTODO}

Caracterização do estudo

Este estudo, envolvendo variáveis antropometricas e de aptidão física pode ser considerado segundo Thomas e Nelson (2002), como uma pesquisa descritiva, comparativa e de corte transversal.

Grupos de Convivência Selecionados

O município de Marechal Candido Rondon - PR possui 14 Grupos de Convivência de Idosos, dos quais foram selecionados para este estudo dois deles: o "Paz e Amor" e o "Amizade". Para seleciona-los foi utilizado como critério de inclusão os seguintes aspectos: a) os dois Grupos estarem localizados na sede do município; b) os encontros serem semanais, pois os demais Grupos do município tem encontros quinzenais; c) maior quantidade de idosos freqüentadores.

\section{AMOSTRA}

A amostra foi composta por 60 sujeitos participantes dos Grupos de Convivência de Idosos, sendo 30 do Grupo Paz e Amor e 30 do Grupo Amizade. A proporção geral em relação ao gênero para o Grupo Amizade foi 76,7\% ( $\mathrm{n}=23)$ feminino e 23,3\% ( $\mathrm{n}=7)$ masculino, já para o Grupo de Convivência de Idosos Paz e Amor foi de 60\% ( $\mathrm{n}=18)$ feminino e 40\% ( $\mathrm{n}=12)$ masculino. Todos os sujeitos participaram voluntariamente do estudo. Desta forma, a amostragem caracterizou-se como não 
probabilística por conveniência.

Todos os participantes deste estudo foram informados dos objetivos e procedimentos do estudo, aqueles que concordaram em participar da pesquisa assinaram ao Termo de Consentimento Livre e Esclarecido. Em acordo com a disposição do Ministério da Saúde Através das normas e diretrizes do Conselho Nacional de Saúde que regulamentam a pesquisa em seres humanos (196/96), este estudo foi avaliado e aprovado pelo Comitê de Ética em Pesquisa da Universidade São Judas Tadeu, registrado Através do parecer consubstanciado, sob processo $n^{\circ}$ 039/2005 em 04 de agosto de 2005. Esta aprovação garantiu o respeito a dignidade humana e o desenvolvimento da pesquisa dentro dos padrões eticos. Instrumentos utilizados.

Os sujeitos do estudo foram submetidos a realização de uma anamnenese Através do Questionário de Prontidão da Atividade Física.

A avaliação antropometrica consistiu na mensuração da estatura, massa corporal, Índice de Massa Corporal (IMC) e circunferência abdominal. A classificação do IMC obedeceu aos critérios da Organização Mundial da Saúde (OMS, 1998) em que considera Baixo Peso indivíduos com índices $<18,5(\mathrm{Kg} / \mathrm{m})$, Normal entre 18,5 a 24,9(Kg/m $\left.{ }^{2}\right)$, Sobrepeso de 25 a $29,9\left(\mathrm{Kg} / \mathrm{m}^{2}\right)$ e Obeso acima de $30\left(\mathrm{Kg} / \mathrm{m}^{2}\right)$. Para classificar os indicadores da Circunferência Abdominal foi adotado os critérios estabelecidos pela OMS (1998) considerando em risco aumentado para valores $>94 \mathrm{~cm}$ no gênero masculino e $>80 \mathrm{~cm}$ no feminino, e muito aumentado para valores $>102 \mathrm{~cm}$ no masculino e $>88 \mathrm{~cm}$ no feminino.

Em relação á aptidão física, os indivíduos/idosos foram submetidos aos seguintes testes selecionados de Matsudo (2005); Rikli e Jones (1999): Forca/resistência de membros superiores (flexão de cotovelo), Forca de membros inferiores (impulsão vertical sem auxilio dos bracos), Potencia aerobia (caminhada de 6 minutos), Flexibilidade (sentar e alcancar), Agilidade (shutle run) e equilíbrio (equilíbrio estatico com controle visual).

\section{ANALISE ESTATÍSTICA}

A tabulação dos dados e analise estatística foi realizada com o programa computadorizado SPSS 12.0.1 for Windows ${ }^{\circledR}$. O tratamento estatístico descritivo foi apresentado mediante medias, desvios padrão, freqüências e percentagens. 
A normalidade das variáveis foi testada Através do teste Kolmogorov-Smirnov, sendo que todas as testadas e analisadas apresentaram distribuição normal. O teste t de Student independente, com nível de significância adotado em $\mathrm{p}<0,05$ foi empregado para verificar diferenças entre os grupos na antropometria e testes de aptidão física.

\section{RESULTADOS}

Com a finalidade de analisar e comparar o perfil antropometrico e de aptidão física dos sujeitos participantes do Grupo de Convivência Paz a Amor e do Grupo Amizade, os resultados são apresentados Através de tabelas.

Sobre as variáveis antropometricas adotadas neste estudo (peso, estatura, IMC e circunferência abdominal), através da Tabela 1 são apresentados os resultados da distribuição media dos sujeitos, divididos e unificados os Grupos de Convivência de Idosos.

Tabela 1 - Distribuicao das medias e desvios padrão do peso, estatura, IMC circunferência abdominal dos sujeitos, por sexo e Grupo de Convivência.

\begin{tabular}{cccccccccc}
\hline & \multicolumn{2}{c}{ PESO $(\mathrm{kg})$} & \multicolumn{2}{c}{ ESTATURA $(\mathrm{m})$} & \multicolumn{2}{c}{ IMC $\left(\mathrm{kg} / \mathbf{m}^{2}\right)$} & \multicolumn{2}{c}{ CIRC. ABD.(cm) } \\
\hline & $\mathrm{M}$ & $\mathrm{F}$ & $\mathrm{M}$ & $\mathrm{F}$ & $\mathrm{M}$ & $\mathrm{F}$ & $\mathrm{M}$ & $\mathrm{F}$ \\
Amiza & 78,34 & 69,40 & 1,71 & 1,59 & 26,86 & 27,41 & 99,07 & 90,33 \\
de & $\pm 9,71$ & $\pm 14,10$ & $\pm 0,06$ & $\pm 0,07$ & $\pm 4,17$ & $\pm 4,52$ & $\pm 11,05$ & $\pm 12,73$ \\
& $\mathrm{n}=7$ & $\mathrm{n}=23$ & $\mathrm{n}=7$ & $\mathrm{n}=23$ & $\mathrm{n}=7$ & $\mathrm{n}=23$ & $\mathrm{~N}=7$ & $\mathrm{n}=23$ \\
& & & & & & & & \\
Paz e & 83,71 & 68,62 & 1,73 & 1,58 & 27,96 & 27,49 & 103,33 & 90,42 \\
Amor & $\pm 12,52$ & $\pm 13,13$ & $\pm 0,05$ & $\pm 0,05$ & $\pm 4,35$ & $\pm 5,06$ & $\pm 9,96$ & $\pm 10,96$ \\
& $\mathrm{n}=12$ & $\mathrm{n}=18$ & $\mathrm{n}=12$ & $\mathrm{n}=18$ & $\mathrm{~N}=12$ & $\mathrm{n}=18$ & $\mathrm{~N}=12$ & $\mathrm{n}=18$ \\
& & & & & & & & \\
Teste t & 0,9729 & 0,1811 & 0,7825 & 0,5119 & 0,5395 & 0,0533 & 0,8648 & 0,0238 \\
P valor & 0,3443 & 0,8572 & 0,4447 & 0,6116 & 0,5966 & 0,9577 & 0,3992 & 0,9811 \\
& & & & & & & & \\
Geral & 81,73 & 69,06 & 1,72 & 1,58 & 27,55 & 27,45 & 101,76 & 90,37 \\
& $\pm 11,59$ & $\pm 13,52$ & $\pm 0,05$ & $\pm 0,07$ & $\pm 4,20$ & $\pm 4,70$ & $\pm 10,29$ & $\pm 11,84$ \\
& $\mathrm{n}=19$ & $\mathrm{n}=41$ & $\mathrm{n}=19$ & $\mathrm{n}=41$ & $\mathrm{~N}=19$ & $\mathrm{n}=41$ & $\mathrm{~N}=19$ & $\mathrm{n}=41$ \\
\hline
\end{tabular}

$\mathrm{M}=$ masculino, $\mathrm{F}=$ feminino. $\mathrm{p}<0,05$.

As tabelas 2 e 3 apresentam os valores médios, desvios padrão e a comparação entre os dois Grupos. 
Tabela 2 - Valores médios e desvios padrão dos sujeitos nos testes de aptidão física das capacidades forca de membros superiores, forca de membros inferiores e flexibilidade.

\begin{tabular}{|c|c|c|c|c|c|c|}
\hline & \multicolumn{2}{|c|}{$\begin{array}{c}\text { Força Mem. Superiores } \\
\text { (rep.) }\end{array}$} & \multicolumn{2}{|c|}{$\begin{array}{c}\text { Força Mem. Inferiores } \\
(\mathrm{cm})\end{array}$} & \multicolumn{2}{|c|}{$\begin{array}{l}\text { Flexibilidade } \\
\text { (cm.) }\end{array}$} \\
\hline$\underset{\mathrm{de}}{\mathrm{Amiza}}$ & $\begin{array}{c}\mathrm{M} \\
16,67 \\
\pm 3,20 \\
\mathrm{n}=7\end{array}$ & $\begin{array}{c}\mathrm{F} \\
15,91 \\
\pm 3,57 \\
\mathrm{n}=23\end{array}$ & $\begin{array}{c}\mathrm{M} \\
22,14 \\
\pm 6,07 \\
\mathrm{n}=7\end{array}$ & $\begin{array}{c}\mathrm{F} \\
14,37 \\
\pm 4,08 \\
\mathrm{~N}=23\end{array}$ & $\begin{array}{c}\mathrm{M} \\
20,79 \\
\pm 6,98 \\
\mathrm{n}=7\end{array}$ & $\begin{array}{c}\mathrm{F} \\
27,22 \\
\pm 6,75 \\
\mathrm{~N}=23\end{array}$ \\
\hline $\begin{array}{l}\text { Paze } \\
\text { Amor }\end{array}$ & $\begin{array}{l}13,17 \\
\pm 2,79 \\
\mathrm{n}=12\end{array}$ & $\begin{array}{l}13,89 \\
=5,25 \\
n=18\end{array}$ & $\begin{array}{l}19,71 \\
\pm 7,63 \\
n=12\end{array}$ & $\begin{array}{l}14,89 \\
\pm 3,43 \\
N=18\end{array}$ & $\begin{array}{c}15,63 \\
\pm 10,73 \\
n=12\end{array}$ & $\begin{array}{l}23,25 \\
\pm 9,13 \\
n=18\end{array}$ \\
\hline $\begin{array}{l}\text { Teste } t \\
P \text { valor }\end{array}$ & $\begin{array}{l}2,502 \\
0,0229\end{array}$ & $\begin{array}{l}1,465 \\
0,1510\end{array}$ & $\begin{array}{l}0,7178 \\
0,4827\end{array}$ & $\begin{array}{l}0,4337 \\
0,6669\end{array}$ & $\begin{array}{l}2,191 \\
0,0370\end{array}$ & $\begin{array}{l}1,602 \\
0,1173\end{array}$ \\
\hline Geral & $\begin{array}{l}14,33 \\
\pm 3,31 \\
\mathrm{n}=19\end{array}$ & $\begin{array}{l}15,02 \\
\pm 4,44 \\
\mathrm{n}=41\end{array}$ & $\begin{array}{l}20,61 \\
\pm 7,02 \\
n=19\end{array}$ & $\begin{array}{l}14,60 \\
\pm 3,77 \\
N=41\end{array}$ & $\begin{array}{l}17,53 \\
\pm 9,65 \\
\mathrm{n}=19\end{array}$ & $\begin{array}{l}25,48 \\
\pm 8,03 \\
\mathrm{~N}=41\end{array}$ \\
\hline
\end{tabular}

Através da Tabela 2 verificou-se que em relação ao teste de força de membros superiores para os homens a media do Grupo Amizade foi superior comparada ao Grupo Paz e Amor, apresentando diferença estatística significativa. Também houve diferença na comparação da flexibilidade masculina ( $\mathrm{p}=0,0370$ ), onde o Grupo Amizade apresentou medias superiores ao Grupo Paz e Amor. 
Tabela 3 - Valores médios e desvios padrão dos sujeitos nos testes de aptidão física das capacidades resistência cardiorrespiratoria, agilidade e equilíbrio.

\begin{tabular}{|c|c|c|c|c|c|c|}
\hline & \multicolumn{2}{|c|}{$\begin{array}{c}\text { Resistência } \\
\text { Cardiorrespiratória (m) }\end{array}$} & \multicolumn{2}{|c|}{$\begin{array}{l}\text { Agilidade } \\
\text { (seg.) }\end{array}$} & \multicolumn{2}{|c|}{$\begin{array}{l}\text { Equilíbrio } \\
\text { (seg.) }\end{array}$} \\
\hline \multirow{4}{*}{$\mathrm{e}_{\mathrm{e}}^{\mathrm{Amizad}}$} & $\mathrm{M}$ & $\mathrm{F}$ & $\mathrm{M}$ & F & $\mathrm{M}$ & F \\
\hline & 547,86 & 486,96 & 16,62 & 19,59 & 22,81 & 17,69 \\
\hline & $\pm 68,85$ & $\pm 43,69$ & $\pm 2,52$ & $\pm 2,33$ & $=9,30$ & $\pm 8,39$ \\
\hline & $N=7$ & $\mathrm{n}=23$ & $\mathrm{n}=7$ & $\mathrm{~N}=23$ & $\mathrm{n}=7$ & $\mathrm{~N}=23$ \\
\hline Paze & 526,67 & 528,89 & 17,89 & 18,91 & 20,51 & 20,30 \\
\hline \multirow[t]{2}{*}{ Amor } & $\pm 49,37$ & $\pm 64,80$ & $\pm 2,28$ & $=2,57$ & $\pm 10,32$ & $\pm 8,95$ \\
\hline & $\mathrm{n}=12$ & $\mathrm{n}=18$ & $\mathrm{n}=12$ & $\mathrm{~N}=18$ & $\mathrm{n}=12$ & $n=18$ \\
\hline Teste $t$ & 0,7815 & 2,471 & 1,128 & 0,8865 & 0,4850 & 0,9601 \\
\hline $\mathrm{P}$ valor & 0,4452 & 0,0179 & 0,2750 & 0,3808 & 0,6339 & 0,3429 \\
\hline Dois & 534,17 & 505,37 & 17,42 & 19,28 & 21,36 & 18,84 \\
\hline \multirow{2}{*}{ Grupos } & $\pm 56,39$ & $\pm 57,25$ & $\pm 2,39$ & $\pm 2,43$ & 9,76 & $\pm 8,63$ \\
\hline & $\mathrm{n}=19$ & $\mathrm{n}=41$ & $\mathrm{n}=19$ & $\mathrm{~N}=41$ & $\mathrm{n}=19$ & $\mathrm{~N}=41$ \\
\hline
\end{tabular}

Foi encontrada diferença significativa na comparação do desempenho do teste de resistência cardiorrespiratoria no gênero feminino, em que o Grupo Paz e Amor teve medias superiores ao Grupo Amizade (Tabela 3). Nas demais comparações entre os Grupos, as variações encontradas não foram consideradas diferentes estatisticamente $(\mathrm{p}<0,05)$.

\section{DISCUSSÃO}

$\mathrm{Na}$ avaliação da antropometria, os sujeitos foram submetidos as seguintes avaliações: peso, estatura, calculo do IMC e circunferência abdominal. Matsudo et. al. (2004) afirmaram que com o passar dos anos o idoso tem uma perda de massa muscular, porem um aumento de gordura corporal, o que repercute no ganho ou perda de peso. Entretanto, evidencia-se que a atividade física regular pode ser um componente fundamental no processo de controle do peso e da gordura corporal durante $o$ envelhecimento (ACSM, 1999; CAPODAGLIO et. al., 2006; KACZOR et. al., 2006; PENEDO e DAHN, 2006).

Segundo Matsudo et. al. (2002) as mudanças que ocorrem nas dimensões do corpo do idoso são uns dos principais indicadores de envelhecimento, principalmente com relação ao peso, estatura e composição corporal. Com as mudanças no peso e na estatura, o IMC, também se modifica com o transcorrer dos anos. Valores de IMC são indicadores práticos recomendáveis para verificar se o idoso se encontra na faixa recomendável de peso. Desta forma, este método poderá relacionar os indicadores com o possível surgimento de certas doenças, principalmente as cardiovasculares e de diabetes. 
Sobre o IMC observa-se Através da Tabela 1 que todos os valores médios apresentados, tanto em relação a gênero ou ao Grupo de Convivência são classificados acima dos recomendáveis pela OMS (1998), ou sejá, encontram-se no estado de pre-obesos. Os valores apresentados equivalem-se ao estudo que teve como objetivo analisar o efeito da atividade física regular sobre parametros antropometricos, desenvolvido por Rogatto e Gobbi (2001), onde constataram que apesar de serem ativos fisicamente a

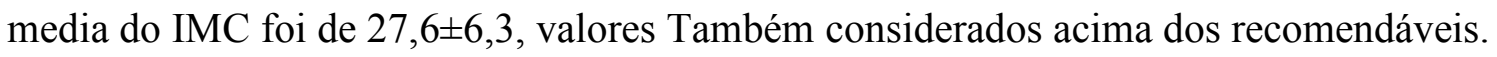

Num estudo em que o objetivo foi verificar a relação entre o índice de aptidão funcional geral e o IMC de mulheres idosas praticantes de atividades físicas, Mazo et. al. (2006) encontraram nos resultados do estudo que as idosas com sobrepeso apresentam um risco aproximadamente 16 vezes maior para terem um índice de aptidão funcional geral em relação àquelas com IMC normal. Desta forma, reforçam a importância do uso de estratégias considerando a pratica da atividade física indispensável e que aliado a uma alimentação adequada para se obter benefício no controle da obesidade.

Os resultados da circunferência abdominal apresentados através da Tabela 1 revelam que as medias em ambos os Grupos de Convivência e gêneros estão com risco elevado para o surgimento de certas doenças, conforme padronização adotada pela OMS, merecendo atenção especial as medias encontradas nos dois Grupos em relação ao gênero feminino, 90,33 cm do Grupo Amizade e 90,42 cm no Grupo Paz e Amor, sendo considerados com risco muito elevado. Para os sujeitos do Grupo de Convivência Paz e Amor com media de 103,33 cm, Também são considerados índices com risco muito elevado. Todos os valores encontrados para a variável circunferência abdominal são superiores se comparados a um estudo desenvolvido por Krause et al. (2006) com mulheres idosas da cidade de Curitiba - PR, em que para o grupo de 60 a 64 anos a media foi de 87,1 cm; 65 a 69 anos foi $87 \mathrm{~cm} ; 70$ a 74 anos foi 86,7 cm; 75 a 79 anos foi $86,9 \mathrm{~cm}$; mais de 80 anos foi de $85,3 \mathrm{~cm}$. Neste estudo, os autores concluíram que os indivíduos que alcançam as idades mais avançadas são aqueles que apresentam menor adiposidade corporal.

De acordo com Costa e Monego (2003) a circunferência da cintura mostra uma relação importante com o risco do aparecimento de eventos cardiovasculares, pela deposição de gordura altamente mobilizável na região do abdómen. Estudos na Educação Física, como os apresentados por Matsudo et al. (2004); Mazo et al. (2005) e Pate et al. (1995) evidenciaram a relação dos índices inadequados com o surgimento de certas doenças. 
Referente a aptidão física no idoso, Matsudo et al. (2004) afirma que e normal os níveis de aptidão física diminuírem no envelhecimento, pois as capacidades físicas não tem o devido potencial que na fase adulta, fato este que poderá exercer influencia sobre o desempenho nas atividades cotidianas do indivíduo. Assim, a manutenção ou melhora da aptidão física será considerado um fator positivo no aspecto físico e funcional do sujeito.

Sobre a capacidade forca muscular, Al Snih (2002) baseado em estudos afirma que com o avanço da idade e característica a diminuição desta capacidade física, o que poderá acarretar na redução da execução de atividades normais do cotidiano como levantar pesos, carregar cargas, puxar objetos ou moveis, dentre outras. Pode-se visualizar Através da Tabela 2 em relação ao teste flexão de cotovelo que e responsável neste estudo para avaliação da capacidade física forca/resistência de membros superiores que os valores médios apresentados em ambos os grupos em relação ao gênero equivalem-se, alem disso, quando apresentados os resultados de forma unificada, a media feminina (15,02 reps.) e superior a masculina (14,33 reps.), contrariando o que a literatura apresenta. De acordo com Spirduso (1995), a força muscular em idosos do sexo masculino normalmente e superior comparada ao sexo feminino, alem disso, nos homens os indicadores de forca tendem a manter-se por mais tempo estável. Na comparação entre os Grupos foi verificado diferença significativa estatisticamente $(p=0,0229)$, pois a media no Grupo Amizade foi de 16,67 repetições, e no Grupo Paz e Amor, 13,17 repetições. Ambos os resultados são inferiores comparados aos padrões de referenda do estudo de Rikli e Jones (1999) com idosos americanos, em que a media para esta variável no teste ficou entre 17,4 a 19 repetições para idosos entre 60 a 74 anos.

Para a capacidade forca de membros inferiores, que foi analisada no presente estudo pela realização do teste impulsão vertical sem auxílio dos braços, mostrou em ambos os gêneros que, apesar das variações entre os Grupos de 2,43 cm para os homens e 0,52 cm para as mulheres, não foram consideradas diferentes estatisticamente.

A flexibilidade segundo Dantas (2003), e a qualidade física responsável pela execução voluntária de um movimento de amplitude angular máxima, por articulacao ou conjunto de articulacoes, dentro dos limites morfologicos, sem risco de provocar lesão. Esta capacidade e influenciada por fatores endogenos, dentre os quais o sexo e considerado um deles, desta forma, as mulheres normalmente são mais flexíveis se comparadas aos homens devido a possuírem tecidos menos densos. Corroborando com a literatura, os resultados médios do gênero feminino foram superiores ao masculino, tanto no Grupo

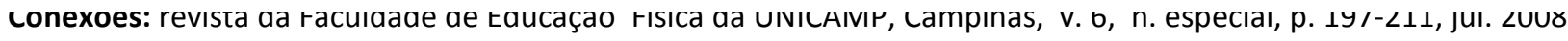
ISSN: $1983-9030$ 
Amizade $(\mathrm{M}=20,79 \mathrm{~cm} ; \mathrm{F}=27,22 \mathrm{~cm})$ quanta no Paz e Amor $(\mathrm{M}=15,63 \mathrm{~cm} ; \mathrm{F}=23,25 \mathrm{~cm})$, alem disso, a diferença encontrada entre os Grupos no gênero masculino de $5,16 \mathrm{~cm}$ foi considerada significativa estatisticamente $(p=0,0370)$. Num estudo realizado por Matsudo et al. (2003) os valores médios apresentados pelas idosas foram de $26,5 \mathrm{~cm}$. Já em outro estudo desenvolvido por Silva Junior et al. (2006) realizado no Projeto Senior para a Vida Ativa, entretanto com a media das idosas ingressantes ao programa nos anos de 2002 a 2004 , a média foi de $24,7 \mathrm{~cm}$.

Em relação à resistência cardiorrespiratoria, Arent et. al.(2000) e Pescatello e Dipietro (1993) atribuem a caminhada como uma atividade ideal para indivíduos idosos, com importantes benefícios por ser um exercício de baixo impacto, que pode ser feita em diferentes intensidades, em qualquer local, envolvendo grandes grupos musculares, contribuindo para aumentar o contato social, principalmente em grupos em risco de isolamento, depressão e dementia. Neste estudo foi utilizado o teste de caminhada de 6 minutos, Através dos resultados apresentados na Tabela 3, verifica-se que a resistência cardiorrespiratoria em geral e superior no desempenho do gênero masculino que apresentou media de $534,17 \pm 56,39$ metres no teste de caminhada de 6 minutos, já a media do gênero feminino foi de $505,37 \pm 57,25$ metres. Se comparados aos valores padrões de referencia para a população na faixa etária de 60 a 64 anos no estudo de Rikli e Jones (1999), verifica-se que os dados do presente estudo apresentaram desempenho inferior nos testes, pois a media para o gênero masculino foi de $613,3 \pm 83,7$ e

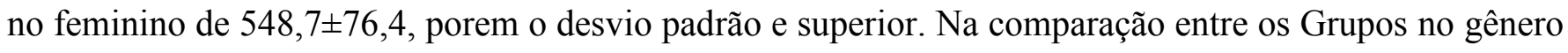
feminino foi constatada diferença significativa estatisticamente $(\mathrm{p}=0,0179)$ na media do desempenho para esta variável.

A agilidade que segundo Zago e Gobbi (2003) e a capacidade exigida em muitas atividades do cotidiano do idoso, como andar desviando de pessoas e obstáculos, locomover-se carregando objetos e andar rapidamente pela casa para atender ao telefone ou campainha. Esta variável foi avaliada Através do teste denominado "Shutle Run". Através dos resultados apresentados no presente estudo verifica-se que os homens apresentaram melhores desempenhos médios em relação as mulheres, pois realizaram o teste em menor tempo. No Grupo de Convivência Amizade a media de execução do teste

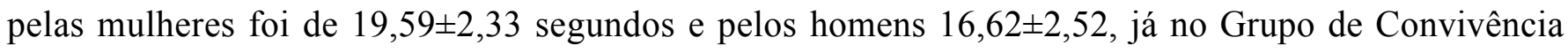

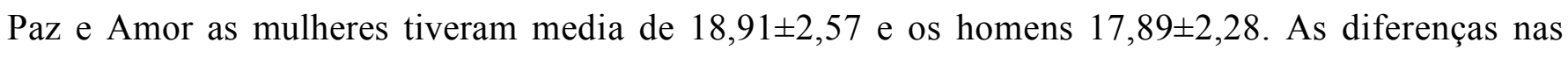
variações da comparação dos resultados entre os Grupos não foram consideradas significativas estatisticamente. 
Os resultados verificados pelos sujeitos do gênero feminino de ambos os Grupos de Convivência são semelhantes aos valores padrões de referencia apresentados por Matsudo et al. (2004) de uma amostra de mulheres fisicamente independentes de São Caetano do Sul, em que a media para a faixa etária de 60 a 69 anos foi de 18,9 $\pm 3,1$ segundos. Quando comparadas com ao desempenho das idosas do estudo realizado por Silva Junior et. al. (2006) que foi de 20,5 $\pm 4,5$ segundos com idade media de $68,3+5,1$ anos, verifica-se que as participantes do trabalho aqui apresentado levaram menos tempo para completar o teste de agilidade proposto, fato que demonstra uma aptidão levemente superior aos valores normativos propostos.

A analise do equilíbrio consiste em importante ferramenta capaz de identificar as limitações no controle dos movimentos, alem de ser útil na determinação do risco de quedas de idosos (DALEY e SPINKS, 2000). Maciel e Guerra (2005) afirmaram que suas alterações no equilíbrio do idoso são consideradas problemas comuns e que leva a importante limitação na realização das atividades da vida diária. De acordo com Hobeika (1999), 65\% dos idosos sofrem frequentemente alguma sensação de tontura ou perda de equilíbrio, alem disso, todos os indivíduos que chegam a esta idade apresentam alguma forma de desequilíbrio. Através da apresentação da analise dos resultados na Tabela 3 verifica-se que mesmo com as variações entre os resultados, não foi encontrada diferença significativa estatisticamente $(\mathrm{p}<0,05)$.

\section{CONSIDERAÇÕES FINAIS}

No aspecto biológico o envelhecimento humano esta relacionado com o surgimento de doenças e declínio das capacidades físicas, interferindo diretamente na realização de tarefas funcionais cotidianas. Sendo assim, e importante realizar uma avaliação física e funcional no idoso com o objetivo de verificar e mostrar as suas limitações e possibilidades frente as ações do seu corpo e os movimentos no dia-a-dia.

Sobre os indicadores antropometricos analisados Através do IMC e circunferência abdominal, verificou-se que, em ambos os Grupos e gêneros, os valores médios apresentados encontram-se acima dos recomendados pela OMS, com indícios de relação com o surgimento de doenças. Sugere-se que palestras de médicos, nutricionistas e profissionais de Educação física poderão incitar reflexões sobre o impacto do tempo sobre variáveis de saúde que podem sofrer interferência positiva pela participação em programas de exercícios. Vale reforçar que apenas palestras relacionadas a saúde, controle de peso, nutrição, hábitos alimentares que auxiliem no processo de informação a população, são importantes, mas não são, isoladamente, capazes de mudar comportamentos. Desta forma, sugere-se 
um processo educativo, capaz de fazer com que os indivíduos tomem consciência de si e dos recursos que possuem para que possam gerenciar o problema.

Os desempenho dos sujeitos nos testes de aptidão física apresentados no presente estudo parecem ser satisfatórios se comparados a literatura, pois corroboram com os resultados de inúmeras pesquisas. Entretanto, a ênfase a ser dada não deve ser geral, mas sim, individual, no sentido de possibilitar ao indivíduo o conhecimento sobre suas capacidades físicas, limites e possibilidades, antes, durante e apos a pratica. Neste sentido, faz-se necessário a avaliação, permitindo ao sujeito conhecer em que nível encontra-se e visualizar se condiz com suas necessidades, fortalecendo a idéia de autonomia dos sujeitos Conclui-se que mesmo com algumas variações de indicadores antropometricos e desempenho nos testes de aptidão física, os Grupos comparados são semelhantes nestas variáveis, provavelmente devido às características gerais da população nos costumes regionais, estilo de vida, historia de vida e hábitos alimentares.

\section{REFERÊNCIAS}

AL SNIH, S.; et al. Handgrip strength and mortality in older Mexican Americans. Journal Am. Geriatr. Soc. v. 50, p. 1250-1256, 2000.

AMERICAN COLLEGE OF SPORTS MEDICINE. ACCSM Programa de condicionamento fisico da ACSM. Sao Paulo: Manole, 1999.

. ACSM's guidelines for exercise testing and prescription. $6^{\text {th }}$ ed. Baltimore: ACSM., 2000.

ARENT, S. M. ; LANDERS, D. M.; ETNIER, J. L. The effects of exercise on mood in older adults: a meta-analytic review. J Aging Phys Act., v. 8 p. 407-430, 2000.

CAPODAGLIO, P.; et al. Long-term strength training for community-dwelling people over 75: impact on muscle function, functional ability and life style. Eur. J. Appl. Physiol., abr. 2006.

COSTA, E. F. A.; MONEGO, E. T. Avaliação geriátrica ampla. Revista da UFG. v. 5, n. 2, dez. 2003.

DALEY, M. J.; SPINKS, W. L. Exercise, mobility and aging. Sports Med. v. 29, n.1, p. 1-12, 2000.

DANTAS, E. H. M. A prática da preparação fisicica. Rio de Janeiro: Shape, 2003.

ELWARD, K. S.; WAGNER, E. H.; LARSON, E. B. Participation by sedentary elderly persons in an exercise promotion session. Fam. Med., 1992, v. 24, n.8, p. 607-612, 1992.

HOBEIKA, C. P. Equilibrium and balance in the elderly. Ear Nose Throat J. v. 78, n. 8, p. 558-562, 1997. 
KACZOR, et al. The effect of aging on anaerobic and aerobic enzyme activities in human skeletal muscle. J. Gerontol. A. Biol. Sci. Med. Sci. v. 61, n. 4, p.339-344, apr. 2006.

KRAUSE, M. P.; et al. Alterações morfológicas relacionadas à idade em mulheres idosas. Revista Brasileira de Cineantropometria e Desempenho Humano, v.8, n.2, p. 73-77, 2006.

MACIEL, A. C. C.; GUERRA, R. O. Prevalência e fatores associados ao déficit de equilíbrio em idosos. Revista Brasileira de Ciência e Movimento. v. 13, n. 1, 2005.

MATSUDO, S. M., Atividade física e envelhecimento: aspectos epidemiológicos. Revista Brasileira de Atividade Física e Saúde, v. 7, n.1, p.2-13, 2001.

MATSUDO, S. M.; BARROS NETO, T. L.; MATSUDO, V. K. R. Perfil antropométrico de mulheres maiores de 50 anos, fisicamente ativas, de acordo com a idade cronológica -evolução de 1 ano. Revista Brasileira de Ciencia e Movimento, v.10, n.2, p.15-26, 2002.

MATSUDO, S. M.; MATSUDO, V. K. R.; BARROS NETO, T. L.; ARAÚJO, T. L. Evolução do perfil neuromotor e capacidade funcional de mulheres fisicamente ativas de acordo com a idade cronológica. Revista Brasileira de Medicina e Esporte. v. 9. n. 6, 2003.

MATSUDO, S. M.; et al. Estudo longitudinal-tracking de 4 anos - da aptidão física de mulheres da maioridade fisicamente ativas. Revista Brasileira de Ciencia e Movimento. v. 12, n. 3, p. 47-52, 2004.

MATSUDO, S. M. Avaliação do idoso: física \& funcional. 2. ed. Londrina: Midiograf, 2005.

MAZO, G. Z. Atividade fisica e qualidade de vida de mulheres idosas. 2003. Tese (Doutorado)Faculdade de Ciências do Desporto e de Educação Física, Universidade do Porto, Porto, Portugal:. 2003

. et al. Tendência a estados depressivos em idosos praticantes de atividade física. Revista Brasileira de Cineantropometria e Desempenho Humano, v. 7, n. 1, p.45-49, 2005.

MAZO, G. Z; ; et al. Aptidão funcional geral e índice de massa corporal de idosas praticantes de atividade fisica. Revista Brasileira de Cineantropometria \& Desempenho Humano. v. 8, n. 4, p. 46-51, 2006.

ORGANIZAÇÂO MUNDIAL DA SAÚDE (OMS) Obesity: preventing and managing the global epidemic. Report of a WHO consultation on obesity. Geneva, 1998.

PATE, R. R.; et al. Physical activity and public health: a recommendation from the Centers for Disease Control and Prevention and the American College of Sports Medicine. Journal of American Medical Association, v. 273, n. 5, p. 402-407, 1995. 
PENEDO, F. J.; DAHN, J. R. Exercise and well-being: a review of mental and physical health benefits associated with physical activity. Curr. Opin. Psychiatry, v. 18, n.2, p. 189-193, 2005.

PESCATELLO, L. S.; DIPIETRO, L. Physical activity in older adults. Sports Med. v. 15, p. 353-364, 1993.

RIKLI, R. E.; JONES, C. J. Development and validation of a functional fitness test for communityresiding older adults. Journal of Aging and Physical Activity, v. 7, p. 129-161, 1999.

ROGATTO, G. P.; GOBBI, S. Efeitos da atividade física regular sobre parâmetros antropométricos e funcionais de mulheres jovens e idosas. Revista Brasileira de Cineantropometria \& Desempenho Humano, v. 3, n. 1, p. 63-69, 2001.

SILVA JÚNIOR, A. P.; et al. Physical fitness indicators for elder women of the Senior Project for Active Life of USJT Universidade São Judas Tadeu. FIEP Bulletin, v. 76, p. 211-214, 2006.

SPIRDUSO, W.W. Physical dimensions of aging. Champaign: Human Kinetics, 1995.

STEWART, A. L.; et al. Physical activity outcomes of Champs II: a physical activity promotion program for older adults. Journal Gerontol. A. Biol. Sci. Med. Sci. v. 56, n. 8, p. m465-470, ago. 2001. STRUCK, B. D.; ROSS, K. M. Health promotion in older adults. Prescribing exercise for the frail and home bound. Geriatrics. v. 61, n.5, p. 22-27.

THOMAS, J. R.; NELSON, J. K. Métodos de pesquisa em atividade física. 3. ed. Porto Alegre: Artmed, 2002.

VALE, R. G. S.; NOVAES, J. S.; DANTAS, E. H. M. Efeitos do treinamento de força e de flexibilidade sobre a autonomia de mulheres senescentes. Revista Brasileira de Ciência e Movimento. v. 13, n. 2, p. 3340.

ZAGO, A. S.; GOBBI, S. Valores normativos de aptidão funcional de mulheres de 60 a 70 anos. Revista Brasileira de Ciência e Movimento; v. 11, n. 2, p.77-86, jun. 2003. 


\section{Arestides Pereira da Silva Junior}

Universidade São Judas Tadeu

Maria Luiza de Jesus Miranda

Universidade São Judas Tadeu

Marília Velardi

Universidade São Judas Tadeu

\section{Referência do artigo:}

\section{ABNT}

JUNIOR, A. P. S., et. al. Perfil antropométrico e de aptidão física de idosos participantes de grupos de convivência. Conexões, v. 6, p. 197-211, 2008.

\section{APA}

Junior, A. P. S., Miranda, M. L. J., \& Velardi, M., (2008). Perfil antropométrico e de aptidão física de idosos participantes de grupos de convivência, 6, 197-211.

\section{VANCOUVER}

Junior APS, Miranda MLJ, Velardi M. Perfil antropométrico e de aptidão física de idosos participantes de grupos de convivência. Conexões, 2008; 6: 197-211. 